PROCEEDINGS OF THE

AMERICAN MATHEMATICAL SOCIETY

Volume 132, Number 4, Pages 1203-1210

S 0002-9939(03)07156-9

Article electronically published on June 23, 2003

\title{
THE INFLUENCE OF DETERMINISTIC NOISE ON EMPIRICAL MEASURES GENERATED BY STATIONARY PROCESSES
}

\author{
YOURI DAVYDOV AND RIČARDAS ZITIKIS
}

(Communicated by Claudia M. Neuhauser)

\begin{abstract}
We consider weak convergence of empirical measures generated by stationary random process $X$ perturbed by deterministic noise $N$. We assume that the noise $N$ has asymptotic distribution. In particular, we demonstrate that if the process $X$ is ergodic, or satisfies some mixing assumptions, then the influence of deterministic noise $N$ on $X$ is the same as it would be if $N$ were stochastic. Such results are of importance when investigating fluctuations and convex rearrangements of stochastic processes.
\end{abstract}

\section{INTRODUCTION}

In many practical and theoretical problems, we encounter stochastic processes of the form

$$
X+N:=\left\{X_{i}+\nu_{i}, i \geq 1\right\},
$$

where $X=\left\{X_{i}, i \geq 1\right\}$ is a stationary stochastic process of interest and $N=$ $\left\{\nu_{i}, i \geq 1\right\}$ is some "noise". Depending on the problem, the noise $N$ can be either random or deterministic. We shall now discuss the two situations in detail.

It is usually assumed in the literature that the noise $N$ is independent of $X$ and is in the form of a sequence of independent and identically distributed random variables. (In this case, $N$ is sometimes called white noise.) Then, for example, assuming that the stochastic process $X$ is ergodic, one proves that, almost surely, the sequence $\left\{Q_{n}, n \geq 1\right\}$ of (random) empirical measures defined by the formula

$$
Q_{n}(B):=\frac{1}{n} \sum_{i=1}^{n} \mathbf{I}\left\{X_{i}+\nu_{i} \in B\right\}, B \in \mathcal{B}(\mathbf{R})
$$

converges weakly to the convolution $\mathcal{L}_{\nu_{1}} * \mathcal{L}_{X_{1}}$; we used $\mathcal{L}_{\nu_{1}}$ and $\mathcal{L}_{X_{1}}$ to denote the marginal probability laws of $X$ and $N$, respectively, and $\mathcal{B}(\mathbf{R})$ to denote the Borel $\sigma$-algebra on the real line $\mathbf{R}$.

The goal of this paper is to demonstrate that the aforementioned statement $Q_{n} \Rightarrow \mathcal{L}_{\nu_{1}} * \mathcal{L}_{X_{1}}$ also remains valid in the case when the noise $N$ is deterministic, that is, when $N=A$, where $A:=\left\{a_{i}, i \geq 1\right\}$ is a deterministic sequence with

Received by the editors February 4, 2002 and, in revised form, December 17, 2002.

2000 Mathematics Subject Classification. Primary 60G10, 60B10; Secondary 60G57.

Key words and phrases. Stationary process, ergodic process, mixing process, empirical measure, weak convergence, strong convergence.

The first author was supported in part by the RFBR Grant 99-01-00112.

The second author was supported in part by an NSERC of Canada individual research grant at the University of Western Ontario. 
some asymptotic law $\mathcal{L}_{A}$ at $+\infty$. As we shall see in Section 2 the limiting measure in this situation remains the same as in the case of random noise; we only have to replace $\mathcal{L}_{\nu_{1}}$ by $\mathcal{L}_{A}$. That is, we shall show in Section 2 that when the noise is deterministic, then $Q_{n} \Rightarrow \mathcal{L}_{A} * \mathcal{L}_{X_{1}}$ either almost surely or in probability, depending on the assumptions.

We conclude the section with an interesting observation that immediately follows from the discussion above. Namely, suppose that the deterministic noise $\left\{a_{i}, i \geq 1\right\}$ has the same asymptotic distribution as the marginal distribution of the random white noise $\left\{\nu_{i}, i \geq 1\right\}$, the latter being independent of $\left\{X_{i}, i \geq 1\right\}$. Then these two - deterministic and random - "noises" $\left\{a_{i}, i \geq 1\right\}$ and $\left\{\nu_{i}, i \geq 1\right\}$ cannot be statistically distinguished. This suggests, for example, that when conducting simulation studies we may choose to work with deterministic sequences having appropriate asymptotic distributions, instead of using random number generators.

\section{MAin RESUlT}

Let $A:=\left\{a_{i}, i \geq 1\right\}$ be a deterministic sequence such that for a probability measure $\mathcal{L}_{A}$ on $\mathcal{B}(\mathbf{R})$ the statement

$$
\frac{1}{n} \sum_{i=1}^{n} \delta_{\left\{a_{i}\right\}} \Rightarrow \mathcal{L}_{A}
$$

holds, where $\delta_{\{z\}}(B)=1$ if $z \in B$ and 0 if $z \notin B$. Furthermore, let $\left\{X_{i}, i \geq 1\right\}$ be a stationary ergodic sequence. Denote the probability law of $X_{1}$ by $\mathcal{L}_{X_{1}}$. Assume that $\mathbf{E}\left(\left|X_{1}\right|\right)<\infty$.

Our goal in this section is to prove weak convergence of the random measures $Q_{n}, n \geq 1$, defined by the formula

$$
Q_{n}(B):=\frac{1}{n} \sum_{i=1}^{n} \mathbf{I}\left\{\left(a_{i}, X_{i}\right) \in B\right\}, B \in \mathcal{B}\left(\mathbf{R}^{2}\right),
$$

as well as to determine the limiting measure $Q$. (Note that the measures $Q_{n}, n \geq 1$, defined above are generalizations of those discussed in Section 1) Specifically, our goal is to present assumptions under which the two statements $Q_{n} \Rightarrow_{P} Q$ and $Q_{n} \Rightarrow_{a . s} Q$ hold. For the completeness of presentation we note that the latter two statements can be understood as follows. Let $d$ denote the Lévy metric. Then, by definition, $Q_{n} \Rightarrow_{P} Q$ if and only if $d\left(Q_{n}, Q\right) \rightarrow_{P} 0$, and $Q_{n} \Rightarrow_{a . s .} Q$ if and only if $d\left(Q_{n}, Q\right) \rightarrow a . s .0$. Now we are in the position to formulate our main result of the paper.

Theorem 2.1. 1) Assume that, for all continuous functions $\phi, \psi: \mathbf{R} \rightarrow \mathbf{R}$ with compact supports and such that $\mathbf{E}\left(\psi\left(X_{1}\right)\right)=0$, the condition

$$
\frac{1}{n} \sum_{i=1}^{n} \phi\left(a_{i}\right) \psi\left(X_{i}\right) \rightarrow 0
$$

holds in probability. Then

$$
Q_{n} \Rightarrow Q:=\mathcal{L}_{A} \times \mathcal{L}_{X_{1}}
$$

in probability. 2) If, however, condition (2.2) holds almost surely, then statement (2.3) holds almost surely. 
If we take any function $h: \mathbf{R} \times \mathbf{R} \rightarrow \mathbf{R}$ which is continuous almost everywhere with respect to the measure $\mathcal{L}_{A} \times \mathcal{L}_{X_{1}}$, then from Theorem 2.1$]$ we immediately obtain that the empirical measures generated by the sequence $\left\{h\left(a_{i}, X_{i}\right), i \geq 1\right\}$ converge weakly to the probability law of $h\left(Y, X_{1}\right)$, where $Y$ is a random variable independent of $X_{1}$ and having the probability law $\mathcal{L}_{Y}=\mathcal{L}_{A}$. The latter statement, in turn, implies that we have $n^{-1} \sum_{i=1}^{n} \delta_{\left\{a_{i}+X_{i}\right\}} \Rightarrow \mathcal{L}_{Y+X_{1}}$ and $n^{-1} \sum_{i=1}^{n} \delta_{\left\{a_{i} X_{i}\right\}} \Rightarrow$ $\mathcal{L}_{Y X_{1}}$ either in probability or almost surely depending on whether assumption (2.2) is satisfied in probability or almost surely. Such results are of importance, for example, in econometrics, time series analysis, and stochastic processes. For more details and further references on applications, we refer, for example, to Bell and Hillmer [1], and Davydov and Zitikis [2], 3].

\section{Notes on ASSUMption (2.2) AND its Verification}

In this section, we discuss a number of sufficient conditions that assure the validity of (2.2) either almost surely or in probability. In particular, in Notes 3.13.7 we present sufficient conditions under which (2.2), and thus (2.3), holds almost surely. In Note 3.8 we give a sufficient condition under which (2.2), and thus (2.3), holds in probability. The conditions we use below are based on some standard and well-known notions such as mixing and weak mixing. Where appropriate, we give precise definitions of those notions in order to avoid possible confusion. For further details on the subject, we refer, for example, to Halmos [4] and Ibragimov and Linnik [5].

Note 3.1. Let $\left\{X_{i}\right\}$ be a stationary and ergodic sequence. Assume that the deterministic sequence $\left\{a_{i}\right\}$ is almost constant, that is, there exist two numbers $\mu \in \mathbf{R}$ and $\gamma>0$ such that

$$
\frac{1}{n} \sum_{i=1}^{n}\left|a_{i}-\mu\right|^{\gamma} \rightarrow 0
$$

We note in passing that when $\gamma=1$, then (3.1) means strong Cesàro convergence of the sequence $\left\{a_{i}\right\}$ to $\mu$.

Statement 3.2. Under the assumptions above, (2.2) holds almost surely.

Proof. We first verify that assumption (3.1) implies $n^{-1} \sum\left|\phi\left(a_{i}\right)-\phi(\mu)\right| \rightarrow 0$ for any function $\phi: \mathbf{R} \rightarrow \mathbf{R}$ specified in the formulation of Theorem 2.1. Furthermore, since the function $\psi$ in (2.2) is bounded, and since the sequence $\left\{X_{i}\right\}$ is ergodic, we have that the sequence $n^{-1} \sum_{i=1}^{n} \psi\left(X_{i}\right)$ converges almost surely to $\mathbf{E}\left(\psi\left(X_{1}\right)\right)$, which is 0 . In view of these observations, we immediately conclude that assumption (2.2) holds almost surely.

Note 3.3. Let the sequence $\left\{X_{i}\right\}$ be stationary and weakly mixing. (The latter assumption is stronger than ergodicity.) We recall (cf. p. 38 of Halmos [4]) that $X$ is weakly mixing if for any $Z$ such that $\mathbf{E}\left(Z^{2}\right)<\infty$ and for any $f$ such that $\mathbf{E}\left(f\left(X_{1}\right)^{2}\right)<\infty$, we have

$$
\frac{1}{n} \sum_{i=1}^{n}\left|\mathbf{E}\left(f\left(X_{i}\right) Z\right)-\mathbf{E}\left(f\left(X_{1}\right)\right) \mathbf{E}(Z)\right| \rightarrow 0 .
$$


Furthermore, assume that $\left\{a_{i}\right\}$ is an almost periodic sequence, that is, for any $\epsilon>0$ there exists a periodic sequence $\left\{a_{i}^{(\epsilon)}\right\}$ such that

$$
\sup _{n \in \mathbf{N}}\left|a_{i}-a_{i}^{(\epsilon)}\right| \leq \epsilon
$$

Statement 3.4. Under the assumptions above, (2.2) holds almost surely.

Proof. Assume for the time being that, for any $\epsilon>0$, assumption (2.2) holds almost surely for the periodic sequence $\left\{a_{i}^{(\epsilon)}\right\}$. More specifically, let the assumption hold on a subset $\Omega^{(\epsilon)} \subseteq \Omega$ of probability one. Assuming without loss of generality that $\epsilon>0$ takes only countable values, we obtain that the statement

$$
\frac{1}{n} \sum_{i=1}^{n} \phi\left(a_{i}^{(\epsilon)}\right) \psi\left(X_{i}\right) \rightarrow 0
$$

holds on the set $\Omega^{0}:=\bigcap_{\epsilon} \Omega^{(\epsilon)}$ for any $\epsilon>0$ from the set of countable values. Note that $\Omega^{0}$ does not depend on $\epsilon>0$ and is of probability one. Before proceeding further, let $c^{(\epsilon)}(\phi)$ denote the supremum of $|\phi(x)-\phi(y)|$ over all $x, y \in \mathbf{R}$ such that $|x-y| \leq \epsilon$. The straightforward inequalities

$$
\begin{aligned}
\limsup _{n \rightarrow \infty}\left|\frac{1}{n} \sum_{i=1}^{n} \phi\left(a_{i}\right) \psi\left(X_{i}\right)\right| & \leq \limsup _{n \rightarrow \infty} \frac{1}{n} \sum_{i=1}^{n}\left|\phi\left(a_{i}\right)-\phi\left(a_{i}^{(\epsilon)}\right)\right|\left|\psi\left(X_{i}\right)\right| \\
& \leq c^{(\epsilon)}(\phi) \lim _{n \rightarrow \infty} \frac{1}{n} \sum_{i=1}^{n}\left|\psi\left(X_{i}\right)\right| \\
& \leq c^{(\epsilon)}(\phi) \mathbf{E}\left(\left|\psi\left(X_{i}\right)\right|\right)
\end{aligned}
$$

hold on $\Omega^{0} \cap \Omega^{e}$, where $\Omega^{e}$ is a subset of $\Omega$ on which $n^{-1} \sum_{i=1}^{n}\left|\psi\left(X_{i}\right)\right|$ converge to $\mathbf{E}\left(\left|\psi\left(X_{1}\right)\right|\right)$. Since $\left\{X_{i}\right\}$ is ergodic, the set $\Omega^{e}$ is of probability one. Thus, the set $\Omega^{0} \cap \Omega^{e}$ is of probability one as well. Now we note that since $\phi$ is uniformly continuous, the constant $c^{(\epsilon)}(\phi)$ converges to 0 when $\epsilon \rightarrow 0$. Thus, the right-hand side of (3.5) also converges to 0 when $\epsilon \rightarrow 0$. Since the left-hand side of (3.5) does not depend on $\epsilon$, we conclude the proof of Statement 3.4 under the assumption imposed at the beginning of this paragraph. We shall now verify that assumption.

Fix any $\epsilon>0$. We want to demonstrate that (3.4) holds on a subset $\Omega^{(\epsilon)} \subseteq \Omega$ of probability one. To simplify the notations, we drop the superscript $(\epsilon)$ and continue working with the notation $\left\{a_{i}\right\}$ instead of $a_{i}^{(\epsilon)}$. Since $\left\{a_{i}\right\}$ is periodic, there exists $p \in \mathbf{N}$ such that $a_{i}=a_{i+p}$ for any $i \geq 1$. With $n=m p$, we continue the proof of (3.4) as follows:

$$
\begin{aligned}
\frac{1}{m p} \sum_{i=1}^{m p} \phi\left(a_{i}\right) \psi\left(X_{i}\right) & =\frac{1}{p} \sum_{i=1}^{p}\left\{\frac{1}{m} \sum_{j=1}^{m} \phi\left(a_{i+(j-1) p}\right) \psi\left(X_{i+(j-1) p}\right)\right\} \\
& =\frac{1}{p} \sum_{i=1}^{p} \phi\left(a_{i}\right)\left\{\frac{1}{m} \sum_{j=1}^{m} \psi\left(X_{i+(j-1) p}\right)\right\} .
\end{aligned}
$$

We see from the equations above that if, for any $i=1, \ldots, p$, the statement

$$
\frac{1}{m} \sum_{j=1}^{m} \psi\left(X_{i+(j-1) p}\right) \rightarrow 0
$$


holds on a subset $\Omega_{i} \subseteq \Omega$ of probability one, then the whole right-hand side of (3.6) converges to 0 on the set $\bigcap_{i=1}^{p} \Omega_{i}$ of probability one. This would conclude the proof of Statement 3.4 We therefore need to verify (3.7).

We shall now demonstrate that, for any $i=1, \ldots, p$, the validity of statement (3.7) follows from the assumption of weak mixing. To see this, we conveniently rewrite (3.2) in the following form: $n^{-1} \sum_{j=1}^{n}\left|b_{j}-b\right| \rightarrow 0$, where $b_{i}:=\mathbf{E}\left(f\left(X_{i}\right) Z\right)$ and $b:=\mathbf{E}\left(f\left(X_{1}\right)\right) \mathbf{E}(Z)$. As noted on p. 38 of Halmos [4], the latter statement implies that $b_{n} \rightarrow b$ when the indices $n \rightarrow \infty$ run through the outside of a set $J \subset \mathbf{N}$ of density 0 . But this obviously implies that $b_{i+(j-1) p} \rightarrow b$ when the indices $j \rightarrow \infty$ are such that $i+(j-1) p$ are outside the set $J$. Using the argument on p. 38 of Halmos [4] once again, we obtain that $n^{-1} \sum_{j=1}^{n}\left|b_{i+(j-1) p}-b\right| \rightarrow 0$. Consequently, the sequence $\left\{X_{i+(j-1) p}, j \geq 1\right\}$ is weakly mixing, and thus ergodic. This concludes the proof of (3.7), and of Statement 3.4 as well.

Note 3.5. Let $\left\{a_{i}\right\}$ be any deterministic sequence satisfying the assumptions of the first paragraph of Section 2. Assume that the process $X=\left\{X_{i}\right\}$ is strongly mixing. For the definition of strong mixing and related results, we refer, for example, to pp. 305-314 of Ibragimov and Linnik [5. Denote the coefficient of strong mixing by $\alpha_{k}$. Furthermore, denote the inverse (i.e. quantile) of the function $x \mapsto P\left(\left|X_{1}\right|>x\right)$ by $K$, and assume that

$$
\int_{0}^{\alpha_{k}} K^{4}(t) d t=O\left(k^{-2}\right), \quad k \rightarrow \infty .
$$

Statement 3.6. Under the assumptions above, (2.2) holds almost surely.

Proof. Denote $b_{i}=\phi\left(a_{i}\right)$ and $Y_{i}=\psi\left(X_{i}\right)$. Note that the two sequences $\left\{b_{i}\right\}$ and $\left\{Y_{i}\right\}$ are bounded. Using relation (7) from Theorem 2 of Rio [6], we obtain the second bound below

$$
\mathbf{P}\left\{\left|\frac{1}{n} \sum_{i=1}^{n} b_{i} Y_{i}\right|>\epsilon\right\} \leq \frac{1}{\epsilon^{4}} \mathbf{E}\left(\left|\frac{1}{n} \sum_{i=1}^{n} b_{i} Y_{i}\right|^{4}\right) \leq \frac{c}{\epsilon^{4} n^{2}},
$$

whereas the first bound above is trivial. Choosing, for example, $\epsilon=n^{-1 / 8}$, we obtain that the right-hand side of (3.9) is of order $O\left(n^{-3 / 2}\right)$. An application of the Borel-Cantelli lemma completes the proof of Statement 3.6

Note 3.7. The strong mixing assumption discussed in Note 3.5 can be replaced by any other assumption on the sequence $X=\left\{X_{i}\right\}$ that assures the validity of the Marcinkiewicz-Zygmund or Rosenthal inequalities. For example, we may consider stationary sequences $X=\left\{X_{i}\right\}$ of associated random variables and use bounds of Shao and Yu [7, instead of Rio [6].

Note 3.8. Let $\left\{a_{i}\right\}$ be any deterministic sequence satisfying the assumptions of the first paragraph of Section 2. Assume that the process $X=\left\{X_{i}\right\}$ is mixing. We recall (cf. p. 37 of Halmos [4]) that $X$ is mixing if for any $Z$ such that $\mathbf{E}\left(Z^{2}\right)<\infty$ and for any $f$ such that $\mathbf{E}\left(f\left(X_{1}\right)^{2}\right)<\infty$, we have the following convergence:

$$
\lim _{n \rightarrow \infty} \mathbf{E}\left(f\left(X_{n}\right) Z\right)=\mathbf{E}\left(f\left(X_{1}\right)\right) \mathbf{E}(Z) .
$$

Statement 3.9. Under the assumptions above, (2.2) holds in probability. 
Proof. For any function $\psi$ satisfying the conditions spelled out in Theorem 2.1, we conclude from (3.10) that $r_{\psi}(k):=\mathbf{E}\left(\psi\left(X_{1+k}\right) \psi\left(X_{1}\right)\right)$ converges to 0 when $k \rightarrow \infty$. In view of this, and since the sequence $\left\{\phi\left(a_{i}\right)\right\}$ is bounded irrespectively of $\left\{a_{i}\right\}$, we obtain that

$$
\mathbf{E}\left\{\frac{1}{n} \sum_{i=1}^{n} \phi\left(a_{i}\right) \psi\left(X_{i}\right)\right\}^{2}=\frac{1}{n^{2}} \sum_{i=1}^{n} \sum_{j=1}^{n} \phi\left(a_{i}\right) \phi\left(a_{j}\right) r_{\psi}(|i-j|) \rightarrow 0 .
$$

Using the Chebyshev inequality, we conclude from (3.11) that (2.2) holds in probability.

\section{Proof of Theorem 2.1}

We shall first prove part 2) (concerning convergence almost surely) and then prove part 1) (concerning convergence in probability). The reason for such an arrangement of the proof of Theorem 2.1 is that we find it easier to prove convergence in probability using the following well-known fact:

A sequence $\left\{\xi_{i}\right\}$ of random variables converges to $\xi$ in probability if and only if for any subsequence $\left\{n^{\prime}\right\} \subseteq\{n\}$ we can find a further subsequence $\left\{n^{\prime \prime}\right\} \subseteq\left\{n^{\prime}\right\}$ such that $\xi_{n^{\prime \prime}}$ converges to $\xi$ almost surely.

Therefore, following this route, we first prove convergence almost surely and then, almost automatically, convergence in probability.

Proof of part 2). We want to find a set $\Omega_{0} \subseteq \Omega$ such that $\mathbf{P}\left\{\Omega_{0}\right\}=1$ and such that, for any $\omega \in \Omega_{0}$,

$$
\iint h(x, y) Q_{n}^{\omega}(d x \times d y) \rightarrow \iint h(x, y) Q(d x \times d y)
$$

for any continuous function $h: \mathbf{R}^{2} \rightarrow \mathbf{R}$ with compact support, where we used the notation

$$
Q_{n}^{\omega}:=\frac{1}{n} \sum \delta_{\left\{\left(a_{i}, X_{i}(\omega)\right)\right\}} .
$$

The above-used class of functions $h$ can certainly be restricted to finite linear combinations of functions of the form $h_{l}=\phi_{l} \psi_{l}$, where the sets $\left\{\phi_{i}\right\}$ and $\left\{\psi_{i}\right\}$ are countable collections of continuous functions $\phi_{l}, \psi_{l}: \mathbf{R} \rightarrow \mathbf{R}$ with compact supports. For example, in both cases we may choose to work with the same set of all trapezoidal functions having rational numbers as their base end-points. In this way, we reduce the proof of statement (4.1) to proving

$$
\iint \phi_{l}(x) \psi_{l}(y) Q_{n}^{\omega}(d x \times d y) \rightarrow \iint \phi_{l}(x) \psi_{l}(y) Q(d x \times d y) .
$$

That is, our goal now is to find a set $\Omega_{0} \subseteq \Omega$ such that $\mathbf{P}\left\{\Omega_{0}\right\}=1$ and such that, for all functions $\phi_{l}$ and $\psi_{l}$ specified above, statement (4.2) holds whenever $\omega \in \Omega_{0}$. 
To this end, we proceed with the following equalities:

$$
\begin{aligned}
\iint \phi_{l}(x) \psi_{l}(y) Q_{n}^{\omega}(d x \times d y)= & \frac{1}{n} \sum_{i=1}^{n} \phi_{l}\left(a_{i}\right) \psi_{l}\left(X_{i}(\omega)\right) \\
= & \frac{1}{n} \sum_{i=1}^{n} \phi_{l}\left(a_{i}\right)\left\{\psi_{l}\left(X_{i}(\omega)\right)-\mathbf{E}\left(\psi_{l}\left(X_{1}\right)\right)\right\} \\
& +\left\{\frac{1}{n} \sum_{i=1}^{n} \phi_{l}\left(a_{i}\right)-\int \phi_{l}(x) \mathcal{L}_{A}(d x)\right\} \mathbf{E}\left(\psi_{l}\left(X_{1}\right)\right) \\
& +\int \phi_{l}(x) \mathcal{L}_{A}(d x) \mathbf{E}\left(\psi_{l}\left(X_{1}\right)\right) \\
= & : A_{n}+B_{n}+C .
\end{aligned}
$$

Obviously, the third summand $C$ in (4.31) equals $\iint \phi_{l}(x) \psi_{l}(y) Q(d x \times d y)$ since $\mathcal{L}_{A} \times \mathcal{L}_{X_{1}}=Q$. Thus, in order to complete the second part of Theorem 2.1 we need to prove that $A_{n}$ and $B_{n}$ converge to 0 . In view of assumption (2.1), the quantity $B_{n}$ converges to 0 . As for the random variable $A_{n}$, we want to demonstrate that there exists a set $\Omega_{0} \subseteq \Omega$ such that $\mathbf{P}\left\{\Omega_{0}\right\}=1$ and such that, for all functions $\phi_{l}$ and $\psi_{l}$ as specified above, the statement

$$
\frac{1}{n} \sum_{i=1}^{n} \phi_{l}\left(a_{i}\right) \psi_{l}^{*}\left(X_{i}(\omega)\right) \rightarrow 0
$$

holds whenever $\omega \in \Omega_{0}$, where we used the notation $\psi_{l}^{*}(x):=\psi_{l}(x)-\mathbf{E}\left(\psi_{l}\left(X_{1}\right)\right)$. (Note that $\mathbf{E}\left(\psi_{l}^{*}\left(X_{1}\right)\right)=0$.) We define the desired set $\Omega_{0}$ as follows. For any pair $\left(\phi_{l}, \psi_{l}\right)$, we construct a set $\Omega_{l} \subseteq \Omega$ such that $\mathbf{P}\left\{\Omega_{l}\right\}=1$ and such that statement (4.4) holds for all $\omega \in \Omega_{l}$. Taking $\Omega_{0}:=\bigcap_{l \in \mathbf{N}} \Omega_{l}$, we obtain the desired $\Omega_{0}$. This finishes the proof of part 2) of Theorem 2.1.

Proof of part 1). Obviously, for any subsequence $\left\{n^{\prime}\right\}$ and for each pair $\left(\phi_{l}, \psi_{l}\right)$ we can find a further subsequence $\left\{n^{\prime \prime}(l)\right\} \subseteq\left\{n^{\prime}\right\}$ such that (2.3) holds. The problem with this is that the just-obtained subsequence $\left\{n^{\prime \prime}(l)\right\}$ depends on $l$, whereas we want to have a subsequence $\left\{n^{\prime \prime}\right\}$ that would be independent of the value of $l \in$ N. The latter goal, however, can easily be achieved by employing the classical diagonalization process. Thus, we can now claim that there exist a sequence $\left\{n^{\prime \prime}\right\}$ and a subset $\Omega_{0} \subset \Omega$ having probability one such that, for all $l \in \mathbf{N}$,

$$
\frac{1}{n^{\prime \prime}} \sum_{i=1}^{n^{\prime \prime}} \phi_{l}\left(a_{i}\right) \psi_{l}^{*}\left(X_{i}(\omega)\right) \rightarrow 0
$$

for all $\omega \in \Omega_{0}$. Applying the already-proved second part of Theorem 2.1, we obtain from (4.5) that

$$
Q_{n^{\prime \prime}} \Rightarrow_{a . s .} Q \text {. }
$$

This finishes the proof of part 1). The proof of the entire Theorem 2.1 is also complete.

\section{ACKNOWLEDGEMENTS}

Our sincere thanks are due to Michael I. Gordin and Michael A. Lifshits for their thought-provoking comments and suggestions that influenced this paper and our view on the subject as well. 


\section{REFERENCES}

1. W. R. Bell and S. C. Hillmer, Issues involved with the seasonal adjustment of economic time series. Journal of Business and Economic Statistics, 20 (2002), 98-127.

2. Y. Davydov and R. Zitikis, Convex rearrangements of random elements. Asymptotic Methods in Stochastics - A Volume in Honour of Miklós Csörgő on the Occasion of His 70th Birthday (Eds. L. Horváth and B. Szyszkowicz), Fields Inst. Commun., Amer. Math. Soc., Providence, RI, 2003.

3. Y. Davydov and R. Zitikis, Convergence of generalized Lorenz curves based on stationary ergodic random sequences with deterministic noise. Statist. Probab. Lett. 59 (2002), 329340.

4. P. R. Halmos, Lectures on Ergodic Theory. Chelsea Publishing Co., New York, 1960.

5. I. A. Ibragimov and Yu. V. Linnik, Independent and Stationary Sequences of Random Variables. Wolters-Noordhoff Publishing, Groningen, 1971. MR 48:1287

6. E. Rio, Inégalités de moments pour les suites stationnaires et fortement mélangeantes. C. R. Acad. Sci. Paris Série I Math. 318 (1994), 355-360. MR 95a:60050

7. Q.-M. Shao and $\mathrm{H}$. Yu, Weak convergence for weighted empirical processes of dependent sequences. Ann. Probab. 24 (1996), 2098-2127. MR 97k:60095

Laboratoire de Mathématiques Appliquées, Université des Sciences et Technologies de Lille, CNRS-Fre 2222, 59655 Villeneuve D'AscQ Cedex, France

E-mail address: Youri.Davydov@univ-lille1.fr

Department of Statistical and Actuarial Sciences, University of Western Ontario, London, Ontario, Canada N6A 5B7

E-mail address: zitikis@stats.uwo.ca 